\title{
O uso do Sistema de Frequência Modulada por crianças e adolescentes atendidos em serviço de saúde auditiva
}

\author{
The use of system of Frequency Modulation by children and \\ adolescents from a hearing health care service
}

Débora Ruttke von Saltiél ${ }^{1}$ (D), Adriane Ribeiro Teixeira ${ }^{1,2}$ (1), Sady Selaimen da Costa ${ }^{1,3}$ (1)

\section{RESUMO}

Objetivo: Analisar o uso e os motivos para o não uso do Sistema de Frequência Modulada (FM) por crianças e adolescentes com perda auditiva e usuários de aparelhos de amplificação sonora individual (AASI) e/ou com implante coclear (IC). Métodos: Foi aplicado um questionário em forma de entrevista, por meio de contato telefônico. As questões foram retiradas do questionário FM Listening Evaluation for Children, traduzido e adaptado para a língua portuguesa por Jacob et al. (2010) e denominado Avaliação do Sistema FM, bem como outras três perguntas elaboradas pelos pesquisadores. Resultados: Foram entrevistados 87 pais/responsáveis, verificando-se que o sistema FM era utilizadopor menos da metade da amostra. Quanto à mediana de uso de horas diárias do sistema, observou-se diferença nos pacientes com FM no IC, que usavam o dispositivo por maior número de horas diárias, do que os usuários de AASI. Igualmente apresentou diferença estatística o principal motivo para onão uso, relacionado ao fato de os pacientes terem recebido novos AASI e/ou IC incompatíveis com a tecnologia obtida anteriormente. Ainda, os entrevistados gostariam que lhes fossem fornecidas melhores orientações. Conclusão: a maior parte dos pacientes não utiliza o sistema FM, sendo o principal motivo o uso de novos AASI e/ou IC. Os pacientes com IC usam o FM de forma mais efetiva (diariamente).

Palavras-chave: Perda auditiva; Auxiliares de audição; Implante coclear; Ruído; Criança; Aprendizagem

\begin{abstract}
Purpose: To analyze de use and reasons for not using of FM systems by children and adolescents with hearing loss and users of hearing aids (HA) and or cochlear implant (CI). Methods: A questionnaire applied in the form of an a interview through telephone contact was used. The questions were removed from the questionnaire "FM Listening Evaluation for children", translated and adapted for Portuguese language by Jacob et al. (2010) and named "Avaliação do Sistema FM", and three other questions prepared by the researchers. Results: 87 parents/guardians were interviewed, and it was verified that less than half of the sample used the FM system. As for the median use of the FM systems daily hours, there was a statistical difference in patients with FM in CI, Who used the device for a greater number of daily hours than hearing AID users. The main reason for non-use also presented statistical difference to the fact that patients have received new hearing aids and/or CI incompatible with previously obtained. Also, respondents would like the guidance provided improved. Conclusion: Most patients did not use the FM system, the main reason being the usage of new hearing aids and/or CI. Patients with CI use FM more effectively (daily).
\end{abstract}

Keywords: Hearing loss; Hearing aids; Cochlear implant; Noise; Child; Learning

\footnotetext{
Trabalho realizado no Hospital de Clínicas de Porto Alegre - HCPA - Porto Alegre (RS), Brasil.

${ }^{1}$ Serviço de Fonoaudiologia, Hospital de Clínicas de Porto Alegre - HCPA - Porto Alegre (RS), Brasil.

${ }^{2}$ Curso de Fonoaudiologia, Departamento de Saúde e Comunicação Humana, Universidade Federal do Rio Grande do Sul - UFRGS - Porto Alegre (RS), Brasil. ${ }^{3}$ Curso de Medicina, Departamento de Oftalmologia e Otorrinolaringologia, Universidade Federal do Rio Grande do Sul - UFRGS - Porto Alegre (RS), Brasil.

Conflito de interesses: Não.

Contribuição dos autores: DRS participou da idealização do estudo, coleta, análise e interpretação dos dados e redação do artigo; ART participou da idealização do estudo, coleta, análise e interpretação dos dados e redação do artigo; SSC participou da idealização e orientação do estudo.

Financiamento: Nada a declarar.

Autor correspondente: Débora Ruttke von Saltiél. E-mail: deborasaltiel@gmail.com

Recebido: Março 14, 2021; Aceito: Outubro 24, 2021
} 


\section{INTRODUÇÃO}

O processo de habilitação ou reabilitação da pessoa com perda auditiva tem como um dos objetivos reduzir os efeitos negativos da deficiência auditiva na comunicação. Tal processo inicia com indicação, seleção e adaptação de aparelhos de amplificação sonora individual (AASI). Atualmente, observamse muitos avanços tecnológicos, tais como o uso de algoritmos, que suprimem parcialmente o ruído ambiental, e de microfones direcionais, que possibilitam a captação de sons de determinada direção. Constata-se, contudo, que os AASI possuem limitações, principalmente quanto a otimizar a relação sinal/ruído de modo adequado para o deficiente auditivo, especialmente quando a fonte sonora está distante ${ }^{(1)}$. O ruído de fundo (competitivo) atrapalha a comunicação e pode gerar prejuízos, tais como cansaço, em razão do maior esforço auditivo, e problemas para a aprendizagem, devido à perda de parte do conteúdo, ou ao recebimento de uma mensagem alterada ${ }^{(2)}$.

Para melhorar a compreensão do sinal/fala em ambientes ruidosos, reverberantes e quando a fonte sonora está distante, existe o Sistema de Frequência Modulada (FM), que é um dispositivo de acessibilidade auditiva(3), utilizado como complemento ao aparelho de amplificação sonora individual (AASI), ou ao implante coclear (IC). Seu uso pode resultar em melhor percepção no reconhecimento de fala para adultos e crianças com e sem perda auditiva ${ }^{(4)}$.

No Brasil, a Portaria n ${ }^{\circ} 1.274$ de 25 de junho de 2013 incluiu o sistema FM na tabela de procedimentos, medicamentos, órteses, próteses e materiais especiais do Sistema Único de Saúde (SUS). De acordo com aportaria, para receber o dispositivo, o paciente deve ter idade mínima de 5 anos e máxima de 17 anos, apresentar deficiência auditiva sensorioneural de grau leve, moderado, severo ou profundo, estar matriculado no ensino fundamental I ou II e/ou no ensino médio. Tal indicação se deve ao fato de que, durante o processo educacional, o aluno com perda auditiva precisa escutar a voz do professor, mesmo em ambientes ruidosos, reverberantes ou em maior distância ${ }^{(3,5)}$. Alguns autores consideram esse sistema uma importante ferramenta educacional ${ }^{(6)}$.

Em 2020, foi publicada nova portaria do Ministério da Saúde (MS) (Portaria n³, de 19 de fevereiro de 2020), com vistas a ampliar o uso de Sistema FM para indivíduos com deficiência auditiva que estejam frequentando qualquer nível acadêmico, independentemente da idade ${ }^{(7)}$, visando à melhora das condições de audibilidade e, consequentemente, a possibilidade de aprendizagem.

O uso do Sistema FM envolve a adequada orientação de pais e professores, considerando-se que a família é fundamental para o processo de adaptação de auxiliares de audição e para o desenvolvimento da linguagem. Os professores passam muito tempo com as crianças na escola e é importante que conheçam o manuseio e o uso do dispositivo para que os alunos tenham uma clara recepção da mensagem oral, passo inicial para o processamento da informação e o decorrente aprendizado escolar $^{(8)}$. Para alguns autores, a orientação deveria se estender a toda a equipe escolar, incluindo professores de apoio, equipe especializada e colegas do usuário, uma vez que o receio em relação ao manuseio e aos cuidados pode gerar o não uso ou o uso parcial do dispositivo na escola ${ }^{(6)}$.

Tendo em vista os benefícios do Sistema FM para a recepção dos estímulos auditivos e o consequente desenvolvimento da linguagem e da aprendizagem, bem como possibilidade de sua concessão por programas de saúde auditiva credenciados no Ministério da Saúde, torna-se necessário investigar o uso desse dispositivo por crianças e adolescentes que, por meio do sistema público, o recebam juntamente com o AASI ou IC. O objetivo do presente estudo foi analisar o uso e os motivos do não uso do Sistema FM por crianças e adolescentes com perda auditiva.

\section{MÉTODOS}

O estudo teve delineamento observacional, analítico e transversal Foi aprovado pelo Comitê de Ética em Pesquisa (CEP) do Hospital de Clínicas de Porto Alegre - HCPA, sob o parecer número 2.140.611. Uma vez que a coleta de dados foi realizada por meio telefônico, e de acordo com a autorização do CEP, a parte inicial da ligação telefônica equivalia ao Termo de Consentimento Livre e Esclarecido (TCLE). Somente após a leitura do texto com esclarecimentos referentes à pesquisa e ao aceite por parte dos pais das crianças e adolescentes, a entrevista era realizada.

Constituíram a amostra crianças e adolescentes com perda auditiva bilateral e usuários de prótese auditiva e/ou implante coclear, que receberam o Sistema FM na instituição, de acordo com os critérios para prescrição determinados pela Portaria1.274. Os pacientes foram identificados por meio de consulta ao livro de protocolo. Posteriormente, realizou-se consulta aos prontuários eletrônicos, para verificação de dados dos pacientes, tais como sexo, idade, tipo e grau de perda auditiva e telefones para contato. Quanto ao grau de perda auditiva, a classificação de escolha dos autores deu-se em concordância com a Organização Mundial da Saúde (OMS), conforme Guia de Orientações na Avaliação Audiológica Básica ${ }^{(9)}$.

Pacientes maiores de 18 anos no momento da entrevista e aqueles cujos pais/responsáveis não atenderam às ligações telefônicas após três tentativas foram excluídos da amostra. A exclusão dos maiores de 18 anos ocorreu em razão do objetivo do estudo, ou seja,o uso do dispositivo por crianças e adolescentes. De acordo com o Estatuto da Criança e do Adolescente (ECA), indivíduos de até 12 anos são considerados crianças e, entre 12 e 18 anos, adolescentes ${ }^{(10)}$.

Para avaliar o uso do Sistema FM em crianças e adolescentes, bem como os motivos para o não uso, utilizou-se parte do instrumento FM Listening Evaluation for Children, criado por Cheryl Johnson, em $2003^{(11)}$, e traduzido e adaptado por Jacob et al. ${ }^{(11)}$. Foram aplicadas as questões referentes ao tópico "Informações sobre o uso do Sistema FM". Para obter informações específicas sobre uso desse recurso na escola, os desafios de sua adaptação e sugestões, os autores do presente estudo elaboraram e incluíram mais três questões.

Considerando-se que muitos pacientes não residem na mesma cidade da instituição onde este estudo foi realizado,optou-se por aplicar o questionário por contato telefônico, seguindo um roteiro criado especialmente para o estudo. Todas as ligações foram feitas dos telefones da instituição, por uma única pesquisadora. Optou-se por realizar a aplicação do instrumento com os pais ou responsáveis devido à idade de parte dos participantes da amostra e à possibilidade de, ao telefone, os pacientes terem dificuldade de compreensão das questões.

A entrevista por telefone obedeceu a um roteiro. Inicialmente, os pais/responsáveis eram questionados sobre o uso diário do Sistema FM. Quando referiam que o paciente não utilizava diariamente, a classificação foi determinada como uso ocasional. Após, questionava-se o número de horas de uso.

Posteriormente, era dada a seguinte explicação:

Vou fazer algumas perguntas sobre algumas situações do dia a dia sem o FM (ou com o FM). Você vai me indicar qual a 
resposta que mais se aproxima da quantidade de vezes que isso acontece com seu filho, em determinada situação. Por exemplo, se a resposta auditiva acontece em determinada situação exposta normalmente, você poderá me indicar " 4 " ou "5". Se ele responde auditivamente para essa situação raramente, você irá indicar " 1 " ou " 2 ". Dessa forma, as opções de respostas variam de 1 a 5 , sendo que, quanto mais frequente ele responde para determinada situação, maior é a pontuação. Um exemplo da resposta que eu desejo de você é: se eu lhe perguntar "Você toma leite?" e você responder 1 (um), isso significa que você quase nunca toma leite, ou se você responder 5 (cinco), significa que você sempre toma leite. Pense antes de responder, tome o tempo que for necessário. Não existe resposta certa ou errada, eu estou interessada em saber o que realmente acontece com seu filho nessas situações. Se eu perguntar alguma coisa que não acontece no dia a dia, me avise, pois podemos marcar a resposta 'não se aplica'. Algumas situações exemplificadas podem não ser relevantes para seu filho nesse momento, mas isso não significa que seu desenvolvimento está atrasado. Como esse questionário é aplicado para crianças de diversas faixas etárias, algumas questões podem não se aplicar, mas é importante que você me relate para que eu possa anotar e verificar seu desenvolvimento nessa situação, em avaliações que serão realizadas futuramente $\mathrm{e}^{(12)}$.

Assim, foram apresentadas para os pais/responsáveis 12 questões. As cinco questões iniciais referiam-se ao manuseio, às condições de funcionamento e ao conforto com o uso da tecnologia, sendo as respostas pontuadas de 1 (raramente) a 5 (normalmente) ou NA, correspondente a 'não se aplica'. Elas estavam assim formuladas:

\section{São fáceis de manipular?}

2. Têm se mantido em boas condições de funcionamento?

3. São confortáveis para o uso da criança?

4. A criança tenta desligar?

\section{Apresentam microfonia (apito)?}

As questões 6 e 7 abordavam as atividades em que foi utilizado o Sistema FM. A pergunta número 6 envolvia oito opções de atividade (recreio, jogos, leitura de histórias, parquinho, caminhadas, terapia fonoaudiológica, shopping, carro), para que fosse indicado em quais delas o paciente utilizava a tecnologia. A pergunta número 7 questionava, entre todas as atividades citadas na questão anterior, em qual o Sistema FM mais tinha ajudado. A questão 8 abordava o conhecimento do entrevistado sobre o maior benefício do Sistema FM. A questão 9 verificava qual fora, na opinião dos pais/responsáveis, a maior mudança notada na criança, devido ao uso da tecnologia.

As questões 10, 11 e 12, elaboradas pelos pesquisadores, objetivavam obter informações sobre o uso do Sistema FM na escola, os desafios para sua adaptação e sugestões sobre o processo de adaptação e uso do dispositivo.

O cálculo do tamanho amostral foi realizado no programa WinPEPI ("Programs for Epidemiologists"), versão 11.43, e baseado no estudo de Alves et al. ${ }^{(13)}$. Para um nível de confiança de $95 \%$, prevalência estimada máxima em $50 \%$ (referente às questões sobre atividade em que o sistema FM é utilizado, facilidade de manipulação, boas condições de funcionamento, conforto, tentativa de desligamento, maior benefício do Sistema FM, mudanças percebidas na criança com o uso do Sistema FM),população que recebeu o Sistema FM (155 crianças e adolescentes), margem de erro de $7 \%$, obteve-se um total mínimo de 87 crianças e adolescentes.

As variáveis numéricas foram descritas por média e desvio padrão, ou mediana e amplitude interquartílica. As variáveis categóricas foram descritas por frequências absolutas e relativas. Para comparar medianas, testes de Mann-Whitney ou de Kruskal-Wallis, em conjunto com o Teste de Dunn, foram aplicados. Na comparação de proporções, os testes Exato de Fisher ou Qui-quadrado, em conjunto com a análise dos resíduos ajustados, foram aplicados. O nível de significância adotado foi de $5 \%(\mathrm{p}<0,05)$ e as análises foram realizadas no programa SPSS versão 21.0.

\section{RESULTADOS}

No período de desenvolvimento da pesquisa, constatou-se que 176 pacientes haviam recebido o Sistema FM na instituição. Deste total, 21 foram excluídos por terem mais de 18 anos de idade no dia do contato e 68 não atenderam as ligações telefônicas após três tentativas, ou o número de contato estava desatualizado. Portanto, a amostra do estudo ficou constituída por 87 pacientes. A média de idade foi $11,9 \pm 2,4$ anos e $56,3 \%$ eram do sexo feminino.

A caracterização auditiva dos pacientes incluídos no estudo evidenciou que $95,4 \%$ deles apresentavam perda auditiva do tipo sensorioneural e o grau da perda auditiva que predominou foi o profundo (Tabela 1).

Verificou-se que, dos 87 pacientes, 39 (44,8\%) crianças/ adolescentes estavam fazendo uso do Sistema FM. Os dados da amostra total dos pacientes que receberam o Sistema FM foram distribuídos correlacionando ao uso, ou não, do dispositivo, considerando a prescrição para uso no AASI ou IC ou AASI e IC, sendo que não houve diferença significativa nesse aspecto

Tabela 1. Caracterização auditiva dos pacientes incluídos no estudo

\begin{tabular}{lc}
\multicolumn{1}{c}{ Variáveis } & $\mathbf{n}=87$ \\
\hline Tipo de perda - OD - $\mathrm{n}(\%)$ & $2(2,3)$ \\
Condutiva & $2(2,3)$ \\
Mista & $83(95,4)$ \\
Sensorioneural & \\
Tipo de perda - OE - $\mathrm{n}(\%)$ & $2(2,3)$ \\
Condutiva & $2(2,3)$ \\
Mista & $83(95,4)$ \\
Sensorioneural & \\
Grau da perda - OD - $\mathrm{n}(\%)$ & $1(1,1)$ \\
Leve & $7(8,0)$ \\
Moderada & $10(11,5)$ \\
Severa & $69(79,3)$ \\
Profunda & \\
Grau da perda - OE - $\mathrm{n}(\%)$ & $2(2,3)$ \\
Leve & $9(10,3)$ \\
Moderada & $6(6,9)$ \\
Severa & $70(80,5)$ \\
Profunda & \\
Perda - $\mathrm{n}(\%)$ & $75(86,2)$ \\
Simétrica & $12(13,8)$ \\
Assimétrica & \\
\hline
\end{tabular}

Legenda: $\mathrm{n}$ = número absoluto; $\mathrm{OD}$ = orelha direita; $\mathrm{OE}$ = orelha esquerda; $\%=$ porcentagem 
avaliado $(\mathrm{p}=1,0)$ (Tabela 2$)$. Os principais motivos do não uso foram incompatibilidade com os auxiliares de audição em uso e problemas técnicos (Figura 1).

Quanto aos dados referentes ao uso diário, ou não, houve semelhança no número de pacientes com AASI ou IC, que referiram usar o dispositivo diariamente. Quanto à mediana de uso de horas diárias do sistema, verificou-se diferença estatística nos pacientes com FM no IC, os quais usavam o dispositivo por maior número de horas diárias, do que os usuários de AASI $(\mathrm{p}=0,002)$ (Tabela 3$)$.

Entre os pacientes que usavam tanto diariamente, quanto ocasionalmente o Sistema FM, foram apresentados dados sobre manuseio, condições de funcionamento, microfonia, conforto e tentativas de desligar o dispositivo. Exceto nas questões relativas às tentativas de desligar e à microfonia, as respostas foram semelhantes entre os usuários diários e ocasionais (Figura 2).

No que se refere aos principais usos do Sistema FM, constatou-se que se relacionaram com a leitura de histórias e com a terapia fonoaudiológica (Figura 3).

A questão 8 abordava o conhecimento dos entrevistados sobre o maior benefício do Sistema FM: 56,4\% responderam que foi para a melhora da compreensão e $20,5 \%$, para diminuição do ruído e amplificação dos sons importantes.

A questão 9 verificava qual fora, na opinião dos pais/ responsáveis, a maior mudança percebida na criança/adolescente, devido ao uso da tecnologia: 38,5\% referiram que 'melhorou a atenção' e 28,2\% denotaram ter sido a 'melhora do aprendizado'.

Após responderem às questões do instrumento adotado, os pais/responsáveis foram inquiridos sobre algumas situações específicas do uso de Sistema FM. No que se refere à escola, do total de entrevistados (87), 66,7\% relataram que houve aceitação de uso no ambiente escolar. Contudo, quando perguntados sobre dificuldades no processo de adaptação, somente $30,2 \%$ responderam que o processo ocorreu sem nenhuma dificuldade. Dentre os fatores desafiadores percebidos no processo, $29,1 \%$ dos entrevistados citaram a 'escola'; 15,1\%, a 'vergonha apresentada pelos pacientes frente ao uso'; 15,1\%, a 'dificuldade de manuseio da tecnologia'. Quando indagados sobre sugestões para a melhora do processo de adaptação do sistema FM, a maior parte dos pais cujos filhos usavam o Sistema FM não tinha sugestões a dar. Entre os pais cujos filhos não usavam o dispositivo, 56\% referiram não ter sugestões; $25 \%$ gostariam que as orientações a eles e aos professores melhorassem, sendo que, neste item, houve diferença significativa entre os pais de pacientes que usavam, ou não, o dispositivo $(p=0,041)$ (Tabela 4).

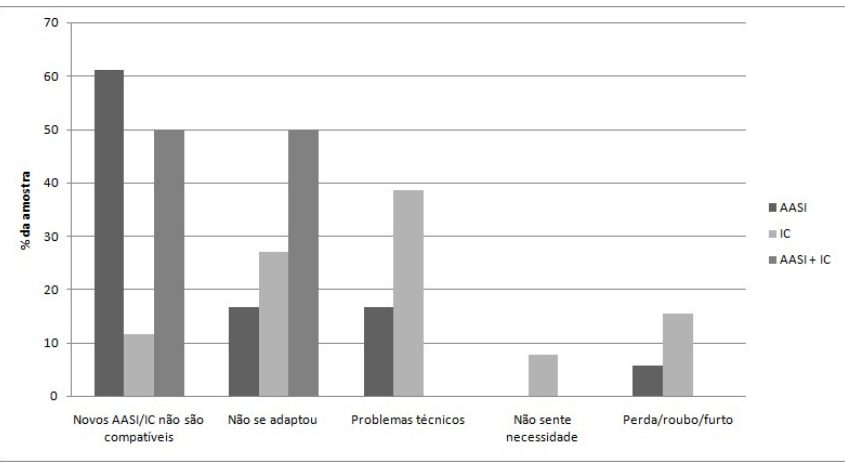

Figura 1. Motivos para o não uso do Sistema de Frequência Modulada Legenda: $\mathrm{AASI}=$ aparelho de amplificação sonora individual; IC = implante coclear; $p$ = associação significativa pelo teste de resíduos ajustados a $5 \%$ de significância

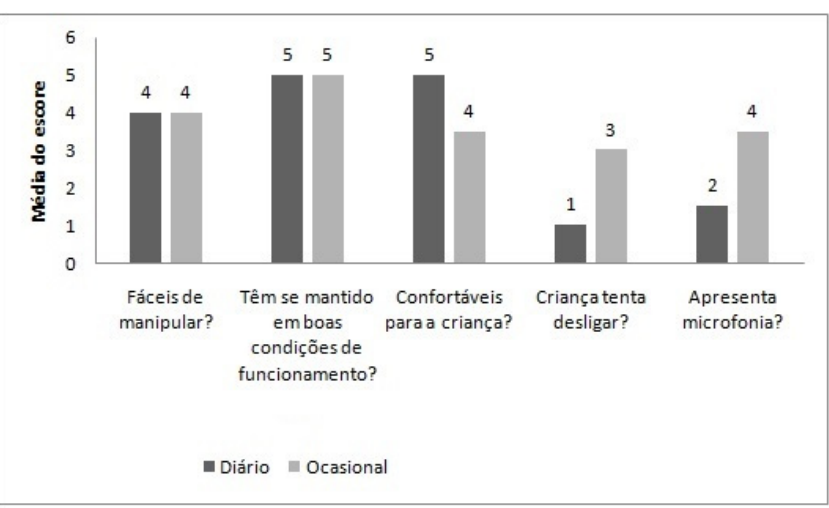

Figura 2. Comparativo dos pacientes que fazem uso diário versus ocasional

Legenda: $1-2=$ raramente; 3 = às vezes; $4-5=$ normalmente

Tabela 2. Associação do uso/não uso na amostra total em relação ao dispositivo onde foi adaptado (aparelho de amplificação sonora individual, implante coclear, ou ambos)

\begin{tabular}{cccc}
\hline Variáveis & FM no AASI $(\mathbf{n = 4 2 )}$ & FM no IC $(\mathbf{n}=\mathbf{4 5})$ & Valor de $\mathbf{p}$ \\
\hline Uso do sistema FM $-\mathrm{n}(\%)$ & & & 1,00 \\
Sim & $19(45,2)$ & $20(44,4)$ & $25(55,6)$ \\
Não & $23(54,8)$ & & \\
\hline
\end{tabular}

Legenda: $\mathrm{AASI}=$ aparelho de amplificação sonora individual; $\mathrm{FM}$ = frequência modulada; $\mathrm{IC}=$ implante coclear; $\mathrm{n}=$ número; \% = porcentagem

Tabela 3. Relato de uso do Sistema de Frequência Modulada em relação à adaptação do dispositivo (aparelho de amplificação sonora individual, implante coclear, ou ambos)

\begin{tabular}{cccc}
\hline Variáveis & FM no AASI $(\mathbf{n = 1 9 )}$ & FM no IC $(\mathbf{n}=\mathbf{2 0})$ & Valor de $\mathbf{p}$ \\
\hline Frequência FM $-\mathrm{n}(\%)$ & & & 1,00 \\
Diariamente & $11(57,9)$ & $12(60,0)$ & $8(40,0)$ \\
Ocasionalmente & $8(42,1)$ & & $5(5-6)$ \\
Horas/dia - md (P25-P75) & $4(4-4)$ & $6(2-12)$ & $0,002^{*}$ \\
Diariamente & $6(3-10)$ & & 0,95 \\
Ocasionalmente & &
\end{tabular}

*Diferença significativa entre os grupos

Legenda: $\mathrm{AASI}=$ aparelho de amplificação sonora individual; $\mathrm{FM}=$ frequência modulada; $\mathrm{IC}=$ implante coclear; $\mathrm{md}=$ mediana; $\mathrm{n}=$ número; \% = percentual; P25 = percentil 25; P75 = percentil 75 
Tabela 4. Sugestões dos pais/responsáveis para manter/ampliar o uso do Sistema de Frequência Modulada

\begin{tabular}{|c|c|c|c|}
\hline Variáveis & Utiliza Sistema FM & Não utiliza Sistema FM & Valor de $p$ \\
\hline Sugestões - n(\%) & & & $0,04^{*}$ \\
\hline Sem sugestões & $29(74,4)$ & $27(56,3)$ & \\
\hline $\begin{array}{l}\text { Melhorar a orientação (pais e } \\
\text { professores) }\end{array}$ & $3(7,7)$ & $12(25,0)^{*}$ & \\
\hline $\begin{array}{l}\text { Melhorar o aparelho (questões } \\
\text { técnicas e estéticas) }\end{array}$ & $6(15,4)$ & $3(6,3)$ & \\
\hline Custo alto da manutenção & $1(2,6)$ & $2(4,2)$ & \\
\hline Tecnologia acompanhar a troca & $0(0,0)$ & $4(8,3)$ & \\
\hline
\end{tabular}

*Associação estatisticamente significativa pelo teste dos resíduos ajustados a $5 \%$ de significância Legenda: $F M=$ frequência modulada

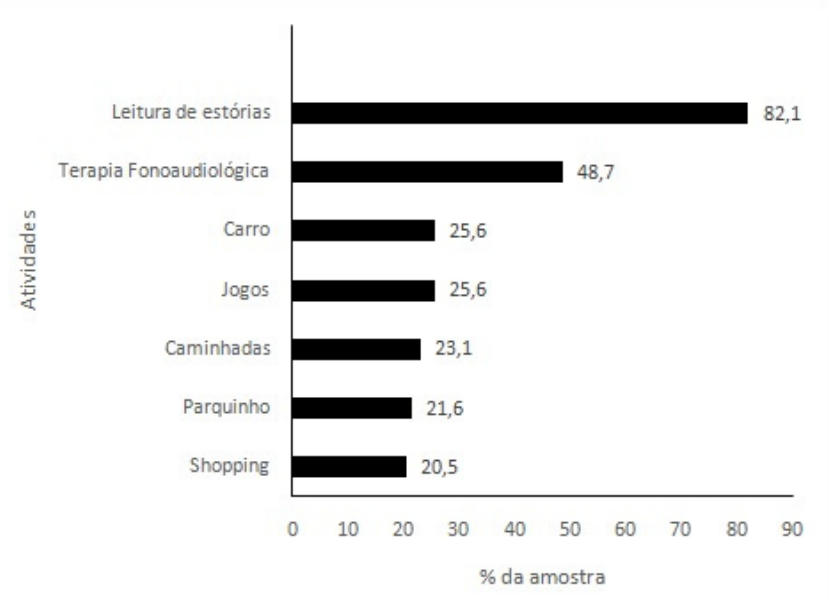

Figura 3. Atividades em que o Sistema de Frequência Modulada é utilizado

\section{DISCUSSÃO}

O presente estudo analisou o uso e os motivos para o não uso da tecnologia do Sistema FM, fornecida pelo SUS, que complementa a adaptação do AASI e/ou IC, com o objetivo de melhorar a recepção e a compreensão da fala em ambientes adversos.

Os resultados obtidos evidenciaram que, aproximadamente, metade dos pacientes incluídos no estudo e que receberam o Sistema FM não o utilizavam, dado que confirma estudos anteriores ${ }^{(14,15)}$. O principal motivo mencionado pelos entrevistados relacionou-se ao fato de terem recebido novos AASI e/ou novos IC incompatíveis com o receptor do Sistema FM anteriormente obtido. O programa de saúde auditiva do SUS prevê a reposição de AASI concedidos, mas não a do Sistema FM. Considerando que muitas crianças não sabem manusear seus dispositivos de amplificação ou zelar por eles, ocorre a redução de sua vida útil. Além disso, entre outros problemas que podem danificar os aparelhos, as crianças participam de atividades de maior risco para os equipamentos, como brincadeiras, e expõem as próteses auditivas a quedas, impactos e umidade. Percebe-se, pois, que criteriosas orientações sobre os cuidados com os dispositivos são tão importantes quanto sua adaptação. Investir em mudanças nessa área configura-se, portanto, como um dos caminhos para minimizar os gastos públicos com as reposições. Estudo realizado no mesmo hospital em que se desenvolveu a presente pesquisa evidenciou que a reposição de AASI em crianças foi feita em tempo médio maior que em adultos e idosos.
Em vista disso, o SUS deveria considerar a possibilidade de manutenção do Sistema FM(16).

Com a publicação da nova portaria pelo Ministério da Saúde, em 2020, houve ampliação da faixa de idade para a concessão do Sistema FM, incluindo indivíduos que frequentam qualquer nível acadêmico ${ }^{(7)}$. A manutenção ou a reposição do Sistema FM em razão de problemas técnicos segue, contudo, não sendo mencionada nos documentos oficiais. Assim, amplia-se a concessão, mas ainda mantém-se o usuário sob o risco de não continuidade de uso se algum problema técnico ocorrer, o que pode ser extremamente prejudicial, especialmente na faixa etária em estudo. Considerando-se a possibilidade de reposição que já existe para os sistemas de amplificação, acredita-se que deveria ser uma opção a ser incluída para os usuários de Sistema FM, uma vez que crianças necessitam de trocas de AASI, especialmente devido a problemas técnicos, necessitando, muitas vezes, de mais de uma reposição ${ }^{(16)}$.

Alguns países que aderiram ao uso do Sistema FM, como Canadá, Lituânia e Jordânia, permitem a reposição. No Canadá, o governo fornece o pagamento de $75 \%$ do valor do sistema a cada três anos. Na Lituânia e na Jordânia, após cinco anos de uso, é possível que o paciente adquira novo equipamento. No entanto, há países, como Brasil, Austria, Dinamarca, França, Alemanha, Suécia, Noruega e Estados Unidos que aderiram ao uso de tal sistema, porém não fazem sua reposição ${ }^{(17)}$.

Desde 25/6/2013, quando foi publicada a Portaria $n^{0} 1.274$, que incluiu o dispositivo FM pessoal como um auxiliar da audição, cabe aos programas de saúde auditiva prescreverem o uso dessa tecnologia, mediante o cumprimento de determinados critérios $^{(18)}$. Respeitar, através de minuciosa avaliação, os critérios de indicação preconizados pela citada portaria é determinante para o êxito da adaptação do Sistema FM, que é individualizada para cada paciente.

Além dos critérios descritos na referida portaria, o fonoaudiólogo pode aferir as condições do Sistema FM utilizando uma ferramenta para verificar sua transparência, ou seja, para verificar suas características eletroacústicas, sendo este um procedimento essencial para o sucesso da adaptação. A 'transparência' foi definida pela American Academy of Audiology (AAA) e permite garantir que a conexão do Sistema FM não altere as configurações de ganho do AASI e que ambos os sinais, FM e microfone do AASI, estejam audíveis. A transparência é atingida quando a entrada de 65 dBNPS para o microfone do FM produz um resultado igual à entrada de 65 dBNPS para o microfone do aparelho de amplificação sonora individual ${ }^{(19)}$. Os dados obtidos na presente pesquisa evidenciaram a necessidade de verificação da transparência do sistema, o que não foi realizado nos pacientes que compuseram a amostra do estudo. Tal fato, associado ao perfil auditivo dos pacientes incluídos na amostra, com elevada prevalência de perda auditiva de grau profundo em ambas as orelhas, evidencia um cenário com predominância de 
pacientes que, com adoção do AASI, têm poucas possibilidades de sucesso, sob o aspecto de percepção auditiva.

Os achados desta pesquisa mostraram que o paciente usuário de IC é aquele que usa o FM de forma mais efetiva (5-6 horas/ dia). Estudos anteriores confirmam esses dados, que evidenciam o FM associado ao IC como um recurso essencial às crianças com deficiência auditiva, principalmente em ambiente escolar ${ }^{(4,14,20-22)}$.

Tais resultados podem ser explicados por várias condições: criteriosa seleção antes da indicação cirúrgica; avaliação interdisciplinar pré-implante; indicação multifatorial, que abrange tipo e grau da perda auditiva, idade, tempo de privação sensorial auditiva; acesso à terapia fonoaudiológica; desenvolvimento global; presença de outros comprometimentos associados à deficiência auditiva; esclarecimento aos pais/responsáveis sobre a importância da audição no desenvolvimento infantil; motivação e participação dos pais/responsáveis no processo de reabilitação da criança ${ }^{(21)}$. Quando a família do implantado mostra-se mais motivada, são geradas atitudes de comunicação favoráveis.

Os entrevistados que referiram o uso da tecnologia diariamente especificaram que isso acontece na escola. $\mathrm{O}$ cenário de uma sala de aula é um exemplo de que fatores como reverberação, distância entre falante e ouvinte, acústica e ruído excessivo podem dificultar a compreensão auditiva e causar prejuízos educacionais. O uso do Sistema FM ameniza esses obstáculos e, consequentemente, suscita oportunidades de melhor aprendizado ${ }^{(1)}$.

Ao serem questionados sobre possíveis desafios no processo de adaptação do Sistema FM, os pais/responsáveis elencaram a escola, a vergonha em fazer uso da tecnologia e as dificuldades de seu manuseio, situações que estão, de certa forma, relacionadas entre si. A dispensação do Sistema FM requer mais do que a avaliação auditiva, faz-se necessário o acompanhamento, por meio de revisões, com o intuito de verificar o uso do sistema e os benefícios a ele relacionados. O acompanhamento dos pacientes que receberam os kits de FM, concedidos pelo SUS, permite a avaliação de seu uso, dos benefícios decorrentes e de eventuais problemas relacionados ao funcionamento do dispositivo.

Dificuldades com a escola constituíram o principal desafio detectado, evidenciando a necessidade de mais integração entre fonoaudiólogo, família, escola, pois as dificuldades podem advir do desconhecimento dos professores em relação à tecnologia do Sistema FM. Estes achados confirmamos de outros autores ${ }^{(8,23-26)}$.

Ressalta-se a importância de disponibilizar materiais que auxiliem os professores no uso dessa tecnologia e a implantação de medidas que possibilitem aos pais/responsáveis a colaboração no processo e, consequentemente, contribuírem para a adaptação ${ }^{(13,15,32)}$.

A 'vergonha' mencionada pelos pacientes quanto ao uso e a 'dificuldade de manuseio' da tecnologia foram igualmente relatadas em estudos anteriores ${ }^{(13,15)}$, nos quais algumas crianças disseram não usar o Sistema FM em sala de aula, apresentando, como justificativa mais frequente, a vergonha perante os outros colegas.

A inserção do paciente com perda auditiva em um ambiente onde poderá ter, mediante convívio com ouvintes, oportunidades de vivenciar experiências auditivas tem favorecido o desenvolvimento da audição, da fala e da linguagem e, certamente, a aprendizagem como um todo. No entanto, para que isso realmente aconteça, é imprescindível o trabalho integrado entre família, escola e fonoaudiólogo.

No que se refere às sugestões dos pais/responsáveis de pacientes que não utilizavam o dispositivo, houve a manifestação sobre a possibilidade de melhor orientação a familiares e professores melhorassem, achados também observados por outros autores ${ }^{(26,27)}$. É cada vez mais comum o ingresso de alunos usuários de AASI ou IC em escolas regulares. Torna-se, pois, necessário empreender ações de preparação dos professores. Treinamento específico para solução de problemas nos sistemas de tecnologia de assistência auditiva e informações educacionais sobre os benefícios do uso da tecnologia assistiva auditiva, em sala de aula, auxiliariam sobremaneira os usuários. Não basta, contudo, uma única consulta para falar sobre a tecnologia. É forçoso promover encontros frequentes que possibilitem o esclarecimento de dúvidas, tanto à família,como aos professores. Um grupo significativo de pais/ responsáveis de usuários do dispositivo identificaram que há benefícios em seu uso. Quanto à atividade em que o Sistema FM mais ajudou, foi indicada a leitura de histórias, achados que concordam com os de outros autores ${ }^{(8,12)}$.

O conhecimento dos entrevistados frente ao maior benefício do Sistema FM também foi identificado em outros estudos ${ }^{(8,13,14)}$ que apresentaram a 'melhora da compreensão' como resultado do uso da tecnologia. Estudos revelam que o uso do Sistema FM favorece a relação positiva do sinal/ruído em mais de $20 \mathrm{dBNA}$, devido à proximidade do microfone - de $6 \mathrm{a} 8 \mathrm{~cm}$ da boca do professor -, influenciando diretamente a melhora da percepção da fala ${ }^{(28-30)}$, aspecto evidenciado pelos pais/ responsáveis entrevistados no presente estudo, que referiram que o uso da tecnologia 'diminui o ruído'.

Ressalta-se que a atenção é precursora do aprendizado e pré-requisito para que ele ocorra. Com a diminuição do ruído, a atenção é favorecida e o desfecho será a melhora da compreensão, refletindo-se no avanço do aprendizado. Achados de outros autores evidenciando que a maior alteração percebida na criança foi a 'melhora na atenção'(8,10,12,13,27,31) e denotando como principal mudança a 'melhora do aprendizado', confirmam os do presente estudo.

Para a consecução do ideário da educação inclusiva, há que se atentar para a formação docente inspirada em princípios da educação para a diversidade, voltando o olhar ao outro e respeitando suas diferenças em sala de aula, para que os alunos com necessidades educacionais especiais alcancem bom desempenho escolar e sintam prazer no ato de aprender ${ }^{(24)}$.

Os resultados positivos não se restringem somente à escola, estão igualmente relacionados aos vários ambientes frequentados pelo usuário. O sistema fornece melhor amplificação, ajuda as habilidades auditivas e dá mais impulso aos aparelhos auditivos, mantendo o usuário focado no falante, permitindo-lhe ter mobilidade e continuar ouvindo.

Dentre as limitações do estudo, destaca-se a impossibilidade em obter informações sobre o uso do Sistema FM em parte dos pacientes que receberam o dispositivo, em função da dificuldade de contato. Apesar de o hospital onde o estudo foi desenvolvido solicitar que os pacientes ou seus familiares mantenham seus telefones atualizados, parte deles não o faz, impedindo que a equipe tenha acesso aos mesmos.

\section{CONCLUSÃO}

O uso do Sistema FM é feito por somente $44,8 \%$ dos pacientes participantes da amostra deste estudo. Aqueles que efetivamente usam a tecnologia diariamente são os usuários de IC.

Os principais motivos para o não uso, informados pelos entrevistados, têm relação com o fato de os pacientes terem recebido novos AASI e/ou IC incompatíveis com a tecnologia antes obtida. 


\section{REFERÊNCIAS}

1. Jacob RTS, Zattoni MQ. Sistemas de frequência modulada. In: Boéchat EM, Menezes PL, Couto CM, Frizzo ACF, Scharlach RC, Anastácio A, editores. Tratado de audiologia. São Paulo: 2015. p. 298-310.

2. Dreossi RCF, Momensohn-Santos TM. A interferência do ruído na aprendizagem. Rev Psicopedag. 2004;21(64):38-47.

3. Santos FR, Delgado-Pinheiro EMC. Relação entre o conhecimento dos professores sobre grau de perda auditiva, dispositivos tecnológicos e estratégias de comunicação. CoDAS. 2018;30(6):e20180037. http:// dx.doi.org/10.1590/2317-1782/20182018037. PMid:30517271.

4. Schafer EC, Thibodeau LM. Speech recognition abilities of adults using cochlear implants with FM Systems. J Am Acad Audiol. 2004;15(10):67891. http://dx.doi.org/10.3766/jaaa.15.10.3. PMid:15646666.

5. Carvalho DS, Pedruzzi CM. Uso do sistema de frequência modulada por escolares com perda auditiva. Distúrb Comun. 2019 Mar 29;31(1):1221. http://dx.doi.org/10.23925/2176-2724.2019v31i1p12-21.

6. Silva Miranda E, Soares Brazorotto J. Facilitadores e barreiras para o uso do Sistema de FM em escolares com deficiência auditiva. Rev CEFAC. 2018;20(5):583-94.

7. Brasil. Ministério da Saúde. Portaria $\mathrm{n}^{\circ} 3$ de 19 de fevereiro de 2020. Torna pública a decisão de ampliar o uso do Sistema de Frequência Modulada Pessoal para indivíduos com deficiência auditiva de qualquer idade matriculados em qualquer nível acadêmico, no âmbito do Sistema Único de Saúde - SUS. Diário Oficial da União [Internet]; Brasília; 2020 [citado em 2021 Mar 14]. Disponível em: https://www.in.gov.br/ web/dou/-/portaria-n-3-de-19-de-fevereiro-de-2020-244302714

8. Rocha BS, Scharlach RC. O uso de Sistema de Frequência Modulada por crianças com perda auditiva: benefício segundo a perspectiva do familiar. CoDAS. 2017;29(6):e20160236. http://dx.doi.org/10.1590/23171782/20172016236. PMid:29069130

9. Conselho Federal de Fonoaudiologia. Guia de orientações na avaliação audiológica básica [Internet]. Brasilia; 2017 [citado em 2021 Mar 14]. Disponível em: http://www.fonoaudiologia.org.br/cffa/wp-content/ uploads/2013/07/Manual-de-Audiologia.pdf

10. Brasil. Lei Federal no 8.069, de 13 de julho de 1990. Diário Oficial da União [Internet]; Brasília; 16 jul 1990 [citado em 2021 Mar 14]. Disponível em: http://www.planalto.gov.br/ccivil_03/leis/18069.htm

11. Gabbard SA. The use of FM Technology for infants and Young children. In: Fabry DA, Johnson CD, editores. ACCESS: achieving clear communication employing sound solutions. Great Britain: Cambrian Printers; 2004. p. 93-9.

12. Jacob RTS, Molina SV, Amorim RB, Bevilacqua MC, Lauris JRP, Moret ALM. FM listeningevaluation for children: adaptação para a língua portuguesa. Rev Bras Educ Espec. 2010;16(3):359-73. http:// dx.doi.org/10.1590/S1413-65382010000300004.

13. Alves LM, Silva BR, Rocha TM, Sales CB, Celeste LC. Avaliação da qualidade de vida em usuários do Sistema de Frequência Modulada. Rev Tecer. 2015;8(15):89-102. http://dx.doi.org/10.15601/1983-7631/ rt.v8n15p89-102.

14. Mezzacasa GP, Aita ADC, Boscolo CC. Desempenho do sistema de frequência modulada em diferentes ambientes comunicativos: percepção dos pais e fonoaudiólogo. In: Costa-Ferreira MID, editor. Reabilitação auditiva: fundamentos e proposições para atuação no Sistema Único de Saúde (SUS). Ribeirão Preto: Book Toy; 2017. p. 377-408.

15. Silva JM, Pizarro LMPV, Tanamati LF. Uso do Sistema FM em implante coclear. CoDAS. 2017;29(1):1-8. http://dx.doi.org/10.1590/23171782/20172016053.

16. Ruschel NL, Bonatto AS, Teixeira AR. Reposição de próteses auditivas em programa de saúde auditiva. Audiol Commun Res. 2019;24:e2025. http://dx.doi.org/10.1590/2317-6431-2018-2025.

17. Brasil. Ministério da Saúde. Sistema de frequência modulada pessoalFM equipamento que possibilita a acessibilidade da criança e/ou jovem com deficiência auditiva na escola [Internet]. Brasilia: Ministério da
Saúde; 2013 [citado em 2021 Mar 14]. Disponível em: http://conitec. gov.br/images/Incorporados/SistemaFM-final.pdf

18. Brasil. Ministério da Saúde. Portaria n ${ }^{\circ} 1.274$, de 25 de junho de 2013. Inclui o Procedimento de Sistema de Frequência Modulada Pessoal (FM) na Tabela de Procedimentos, Medicamentos, Órteses, Próteses e Materiais Especiais (OPM) do Sistema Único de Saúde. Diário Oficial da União; Brasilia; jun 2013 [citado em 2021 Mar 14]. Disponível em: http://bvsms.saude.gov.br/bvs/saudelegis/gm/2013/prt1274_25_06_2013. html

19. American Academy of Audiology. American Academy of Audiology Clinical practice guidelines: remote microphone hearing assistance technologies for children and youth from birth to 21 years. Reston; 2008.

20. Schafer EC, Wolfe J, Lawless T, Stout B. Effects of FM-receiver gain on speech-recognition performance of adults with cochlear implants. Int J Audiol. 2009;48(4):196-203. http://dx.doi.org/10.1080/14992020802572635. PMid:19363720.

21. Iglehart F. Speech perception by students with cochlear implants usings ound-field systems in classrooms. Am J Audiol. 2004;13(1):62-72. http://dx.doi.org/10.1044/1059-0889(2004/009). PMid:15248805.

22. Thibodeau L. Benefits of adaptive FM Systems on speech recognition in noise for listeners who use hearing aids. Am J Audiol. 2010;19(1):36-45. http://dx.doi.org/10.1044/1059-0889(2010/09-0014). PMid:20220201.

23. Leidens B, Boscolo CC, Aita ADC. A formação de professores de escolas regulares para o atendimento de alunos deficientes auditivos. In: Costa-Ferreira MID, editor. Reabilitação auditiva: fundamentos e proposições para atuação no Sistema Único de Saúde (SUS). Ribeirão Preto: Book Toy; 2017. p. 409-28.

24. Gasparetto MERF, Maia SR, Maior EJMI, Nascimento FC, Miranda JR, Ramos CR, et al. Uso de recursos e equipamentos de tecnologia assistiva na educação municipal, estadual e federal tecnológica [Internet]. In: Brasil. Subsecretaria Nacional de Promoção dos Direitos da Pessoa com Deficiência, editor. Tecnologia assistiva. Brasilia; 2009. p. 41-58 [citado em 2021 Mar 14]. Disponível em: https://www.pessoacomdeficiencia. gov.br/app/sites/default/files/publicacoes/livro-tecnologia-assistiva.pdf

25. Delgado-Pinheiro EMC, Antonio FL, Libardi AL, Seno MP. Programa de acompanhamento fonoaudiológico de professores de alunos deficientes auditivos que utilizam a comunicação oral. Distúrb Comun. 2009;21(1):66-77.

26. Libardi AL. Avaliação do site "Curso de sistema de frequência modulada para professores" [dissertação]. Bauru: Universidade de São Paulo; 2012 [citado em 2021 Mar 14]. Disponível em: http://www.teses.usp. br/teses/disponiveis/25/25143/tde-01112012-190309/

27. Nelson LH, Poole B, Muñoz K. Preschool teachers' perception and use of hearing assistive technology in educational settings. Lang Speech Hear Serv Sch. 2013;44(3):239-51. http://dx.doi.org/10.1044/01611461(2013/12-0038). PMid:23843650.

28. Bertachini ALL, Pupo AC, Morettin M, Martinez MAN, Bevilacqua MC, Moret ALM, et al. Frequency Modulation System and speech perception in the classroom: a systematic literature review. CoDAS. 2015;27(3):292-300. http://dx.doi.org/10.1590/2317-1782/20152014103. PMid:26222948.

29. Wolfe J, Schafer EC. Optimizing the benefit of sound processors coupled to personal FM systems. J Am Acad Audiol. 2008;19(8):585-94. http:// dx.doi.org/10.3766/jaaa.19.8.2. PMid:19323350.

30. Jacob RTS, Alves TKM, Moret ALM, Morettin M, Santos LG, Mondelli MFCG. Participation in regular classroom of student with hearing loss: frequency modulation System use. CoDAS. 2014;26(4):308-14. http:// dx.doi.org/10.1590/2317-1782/201420130027. PMid:25211690.

31. Jacob RTS, Almeida MA, Bevilacqua MC. Uso alternativo do sistema de frequência modulada (FM): crianças com dificuldades de aprendizagem e déficit de atenção alternativa. J Bras Fonoaudiol. 2002;3(10):54-9.

32. Balen SA, Brazorotto JS. Uso do Sistema de FM no Ambiente Escolar [Internet]. Natal: SEDIS-UFRN; 2019 [citado em 2021 Mar 14]. Disponível em: https://lais.huol.ufrn.br/wp-content/uploads/2020/03/ sistemaFM_amb_escolar.pdf 